\title{
Research on the Asymmetric S-type Curve Based on the Position
}

\author{
LI Xin;CHEN Le
}

School of Electrical Engineering and Automation,HeFei University of Technology,Hefei 23009,China

E-mail:183678691@qq.com

Key words:Asymmetric ;S-type ;Position;Simulation;Experiment

\begin{abstract}
The acceleration and deceleration section of the symmetric S-type curve are completely symmetrical, which can not meet the different requirements of the actual process and mechanical structure to the acceleration and deceleration section of the motor.Therefore, the asymmetric S-type curve is used to plan the acceleration and deceleration section respectively.First of all, the dichotomy iteration is proposed to solve the problem which has not analytic solutions appearing in the planning process.Secondly, the asymmetric S-type curve is compared with the symmetric S-type curve under different conditions,which indicate that the asymmetric S-type curve is better than the symmetric S-type curve.Finally, the experiment is done on the actual hardware platform. And the actual curve is compared with the theoretical curve, which verify the validity and practicability of the asymmetric S-type curve.
\end{abstract}

\section{Introduction}

There is inevitably position operation in the running of CNC and robots, and the position will put forward some requirements to the dynamic trajectory of the motor ${ }^{[1]}$.Therefore, it is necessary to plan the acceleration and deceleration process of the motor.And this plan must satisfy two conditions: on the one hand,the target position can be reached as quickly and accurately as possible; on the other hand,the operation process of the motor has a certain flexibility and not the impact ${ }^{[2]}$.

Because of the simple calculation and easy control of the trapezoidal curve, the acceleration and deceleration planning is to use this method.But this method has gradually abandoned due to the poor control precision, the sudden change of the accelerated velocity and the impact to the motor.So the S-type curve planning method is proposed, which is more flexible and less impact to the motor than the trapezoidal curve ${ }^{[4,5]}$.However, the acceleration and deceleration section of the traditional S-type curve are symmetrical.That is to say, it only need to plan the process of the acceleration section,the deceleration section can directly copy the planning results of the acceleration section ${ }^{[6]}$.

In the actual industrial site, the mechanical structure and technique have different requirements to the acceleration and deceleration section. Generally, to reduce the impact on the mechanical structure and the motor,the acceleration section should be as smooth as possible and the accelerated velocity is small;to shorten the whole operation time,the deceleration section should be as fast as possible and the decelerated velocity is large.Therefore,the traditional symmetric S-type curve can not satisfy the above requirements, and the parameters can only be selected in accordance with the deceleration section.So this paper presents an asymmetric S-type curve planning method, which use the accelerated velocity(A) and the decelerated velocity(D) to express the acceleration section and the deceleration section of the accelerated velocity. The two parameters are used to meet the different requirements of the acceleration and deceleration section to improve the motor efficiency ${ }^{[7]}$.

\section{Basic Principles and Formulas}

Fig. 1 is the curve of the distance(S), the velocity(V), the accelerated velocity(a) and the jerk(j) in the whole process of the typical seven section S-type curve. The seven section includes the acceleration process, the constant velocity process and the deceleration process. The acceleration process includes the increasing acceleration section, the constant acceleration section and the decreasing acceleration section.And the deceleration process includes the increasing deceleration section, the constant 
deceleration section and the decreasing deceleration section. So it is called the seven section S-type curve. if $\mathrm{A}$ and $\mathrm{D}$ are different, it will cause the different curve between the acceleration process and the deceleration process, the curve is known as asymmetric S-type curve.

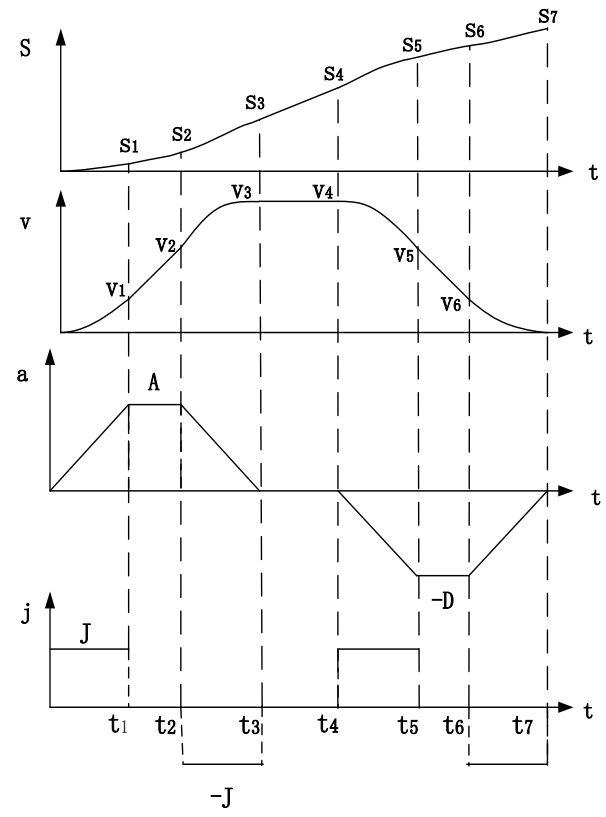

Fig.1 Typical seven section S-type curve

The planning algorithm used in this paper is based on the five input parameters:position(L), maximum velocity(V), maximum accelerated velocity(A), maximum decelerated velocity(D) and jerk $(\mathrm{J})$.In the premise of ensuring the accuracy of $\mathrm{L}$, this paper use asymmetric S-type curve to plan the whole process of movement trajectory.

\section{Asymmetric S-type Curve Plan}

As shown in Fig.1, the increasing acceleration section and the decreasing acceleration section are symmetrical and its running time is the same. That is:

$$
\left\{\begin{array}{l}
T_{1}=T_{3}=\frac{A}{J} \\
T_{5}=T_{7}=\frac{D}{J} \\
\Delta V_{1}=\Delta V_{3}=\frac{A^{2}}{2 J} \\
\Delta V_{5}=\Delta V_{7}=\frac{D^{2}}{2 J}
\end{array}\right.
$$

So planning the whole asymmetric S-type curve process is to judge the existence of the constant acceleration section, the constant velocity section and the constant deceleration section and to calculate the value of $T_{2}, T_{4}, T_{6}$ in the three sections. According to the existence of the three sections, there will be eight types of asymmetric S-type curve .As shown in Fig.2:

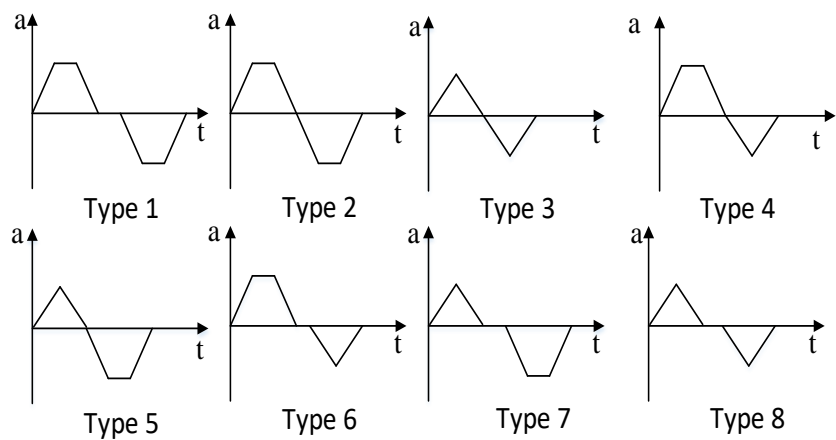

Fig2 8 types of acceleration curves 
The condition for the existence of the constant acceleration section is the command velocity $V_{\max }$ and $A^{2} / J$. If $V_{\text {max }}>A^{2} / J$, there is constant acceleration section; and if $V_{\max } \leq A^{2} / J$, there is no constant acceleration section. The same can be judged whether the existence of the constant deceleration section. The reduction between the sum of distance in the acceleration and deceleration process and command distance can judge the existence of the constant velocity section. So the planning algorithm should plan the velocity and then plan the position ${ }^{[1]}$.

Velocity Plan. 1)If $V_{\max }>A^{2} / J$, there is a constant acceleration section in the acceleration process, and the maximum accelerated velocity $\mathrm{A}$ can be achieved.So the increasing acceleration section time $T_{1}$, the constant acceleration section time $T_{2}$, the decreasing acceleration section time $T_{3}$ are

$$
\left\{\begin{array}{l}
T_{1}=T_{3}=\frac{A}{J} \\
T_{2}=\frac{V \max }{A}-T_{1}
\end{array}\right.
$$

2)If $\mathrm{V}_{\max } \leqslant \mathrm{A}^{2} / \mathrm{J}$, there is no constant acceleration section in the acceleration process, and the A can not reached.So the actual maximum accelerated velocity A' and the times of the three section in the acceleration process are

$$
\left\{\begin{array}{l}
A^{\prime}=\sqrt{V_{\max } J} \\
\mathrm{~T}_{1}=\mathrm{T}_{3}=\frac{\mathrm{A}^{\prime}}{\mathrm{J}} \\
T_{2}=0
\end{array}\right.
$$

3)If $V_{\max }>D^{2} / J$, there is a constant deceleration section in the deceleration process, and the $D$ can be achieved. So the increasing deceleration section time $T_{5}$, the constant deceleration section time $\mathrm{T}_{6}$ and the decreasing deceleration section time $\mathrm{T}_{7}$ are

$$
\left\{\begin{array}{l}
T_{5}=T_{7}=\frac{D}{J} \\
T_{6}=\frac{V_{\max }}{D}-T_{5}
\end{array}\right.
$$

4)If $V_{\max } \leqslant D^{2} / J$, there is no constant deceleration section in the deceleration process, and the $\mathrm{D}$ can not reached. So the actual maximum decelerated velocity D' and the times of the three section in the deceleration process are

$$
\left\{\begin{array}{l}
D^{\prime}=\sqrt{V_{\max } J} \\
T_{5}=T_{7}=\frac{D^{\prime}}{J} \\
T_{6}=0
\end{array}\right.
$$

Distance Plan. $S_{\text {Acc }}$ represents the actual distance of the acceleration section, $S_{\text {Dec }}$ represents the actual distance of the deceleration section, $\mathrm{S}_{\mathrm{Con}}$ represents the actual distance of the constant velocity section, $\mathrm{L}$ represents the distance of the instruction specified.

1)If $S_{\text {Acc }}+S_{\text {Dec }}<L$, there is the constant velocity section, and its time is

$$
T_{4}=\frac{L-S_{\text {Acc }}-S_{\text {Dec }}}{V_{\max }}
$$

The type 1, 6, 7 in Fig2 belong to above conditions, which is simple in the calculation process and the realization.

2)If $S_{\text {Acc }}+S_{\text {Dec }} \geq L$, There is no constant velocity section, that is $T_{4}=0$. Obviously, it will not reach the maximum velocity $\mathrm{V}_{\max }$ specified by the instruction, which requires calculate a new $\mathrm{V}_{\max }$. Without constant velocity section, there will be the following four situations: not exist the constant acceleration and the deceleration section , exist the constant acceleration section but not exist the constant deceleration section, exist the constant deceleration section but not exist the constant acceleration section, exist the constant acceleration section and the constant deceleration section.

3)not exist the constant acceleration section and the constant deceleration section 
$\mathrm{S}_{4 \mathrm{~d}}$ represents the maximum distance in this condition,so

$$
S_{4 \mathrm{~d}}=2 \frac{\min \{A, D\}^{3}}{J^{2}}
$$

If $L \leq S_{4 \mathrm{~d}}$,There is not exist the constant acceleration section and the constant deceleration section.And it is need to calculate the new A and D.

$$
\left\{\begin{array}{l}
A^{\prime}=D^{\prime}=\sqrt[3]{\frac{L J^{2}}{2}} \\
T_{2}=T_{6}=0 \\
T_{1}=T_{3}=T_{5}=T_{7}=\frac{A^{\prime}}{J}
\end{array}\right.
$$

4)exist the constant acceleration section but not exist the constant deceleration section

In this case, there will be $\mathrm{A}<\mathrm{D}$ and the maximum deceleration $\mathrm{D}$ specified by the instruction can not be reached. The $\mathrm{D}^{\prime}$ represents the new maximum deceleration, the $\mathrm{V}_{\max }$ ' represents the new maximum velocity.

$$
\left\{\begin{array}{l}
S_{A c c}=\frac{1}{2}\left(\frac{2 A}{J}+T_{2}\right)\left(\frac{A^{2}}{J}+A T_{2}\right) \\
S_{D e c}=\frac{D^{\prime 3}}{J^{2}} \\
V_{\text {max }}=\frac{A^{2}}{J}+A T_{2}=\frac{D^{\prime 2}}{J} \\
S=S_{A c c}+S_{D e c}=L
\end{array}\right.
$$

It can be seen that there is no analytic solution to the Eq.9, so it is necessary to find another solution.It is obvious that the function $S=S_{A c c}+S_{D e c}$ is a strictly monotone increasing function about the $T_{2}$, so the value of the $T_{2}$ can be solved by the dichotomy iteration, then other parameters can be obtained. The key of the dichotomy iteration is to determine the lower limit value $\mathrm{T}_{2 \text { min }}$ and the upper limit value $\mathrm{T}_{2 \max }$.

When the constant acceleration section does not exist, the $T_{2}$ take the minimum value, $T_{2 \min }=0$; When $D^{\prime}=D$, the $T_{2}$ take the maximum value, $T_{2 \max }=\left(D^{2}-A^{2}\right) /(A J)$. So the range of $T_{2}$ is $\left[0,\left(D^{2}-A^{2}\right) /(A J)\right]$.

Next describe the iterative process

First,select the maximum error of the distance Er, the initial value of the $\mathrm{T}_{2}$ is

$$
T_{2}=\frac{1}{2}\left(T_{2 \min }+T_{2 \max }\right)
$$

Second,use this value to calculate the actual total distance $S=S_{A c c}+S_{D e c}$ in formula set and compare it with the target distance L.If S-L>Er, then the value of the actual total distance $\mathrm{S}$ is too large, and the $T_{2}$ is too large.So take $T_{2 \max }=T_{2}$ and continue the iteration;If $S-L<-E r$, then the value of $\mathrm{S}$ is too small ,and that $\mathrm{T}_{2}$ is too small, take $\mathrm{T}_{2 \min }=\mathrm{T}_{2}$ and continue the iteration.when $|\mathrm{S}-\mathrm{L}|<\mathrm{Er}$, the iteration is stopped, this $\mathrm{T}_{2}$ is evaluated.

5)exist the constant deceleration section but not exist the constant acceleration section

In this case, there will be $\mathrm{A}>\mathrm{D}$ and the maximum acceleration $\mathrm{A}$ specified by the instruction can not be reached.the $A^{\prime}$ presents the new maximum acceleration, the $V_{\max }$ 'represents the new maximum velocity.

$$
\left\{\begin{array}{l}
S_{\text {Dec }}=\frac{1}{2}\left(\frac{2 D}{J}+T_{6}\right)\left(\frac{D^{2}}{J}+D T_{6}\right) \\
S A_{c c}=\frac{A^{\prime 3}}{J^{2}} \\
V_{\text {max }^{\prime}}=\frac{D^{2}}{J}+D T_{6}=\frac{A^{\prime 2}}{J} \\
S=S_{A c c}+S_{D e c}=L
\end{array}\right.
$$


It can be seen that this situation is similar to the above situation that there is no analytic solution. So the dichotomy iteration can be also used to solve this formula set. We know that $\mathrm{T}_{2 \min }=0$, $\mathrm{T} 2 \mathrm{max}=\left(\mathrm{A}^{2}-\mathrm{D}^{2}\right) /(\mathrm{DJ})$. And the whole idea and calculation process are similar to the 2$)$.

6)exist the constant acceleration section and the constant deceleration section.

In this case,it is need to calculate the $\mathrm{T}_{2}, \mathrm{~T}_{6}$ and the new maximum velocity $\mathrm{V}_{\max }$

$$
\left\{\begin{array}{l}
V_{\text {max }}=\frac{A^{2}}{J}+A T_{2}=\frac{D^{2}}{J}+D T_{6} \\
S_{A c c}=\frac{1}{2}\left(\frac{2 A}{J}+T_{2}\right) V_{\text {max }} \\
S_{D e c}=\frac{1}{2}\left(\frac{2 D}{J}+T_{6}\right) V_{\text {max' }} \\
S=S_{A c c}+S_{D e c}=L
\end{array}\right.
$$

Similarly,iterative dichotomy solution is used. It is obvious that $\mathrm{T}_{2 \mathrm{mi}} \mathrm{n}=0, \mathrm{~T}_{2 \max }=$ $\min \left\{\mathrm{V}_{\max } / \mathrm{A}-\mathrm{A} / \mathrm{J}, \mathrm{V}_{\max } / \mathrm{D}-\mathrm{D} / \mathrm{J}\right\}$ And the whole idea and calculation process are similar to the 2 ).

\section{Simulation and Verification}

In order to verify the correctness and practicability of the planning algorithm of the asymmetric S-type velocity curve, this algorithm is simulated by the MATLAB software and this paper compares the asymmetric S-type velocity curve with the traditional symmetric S-type velocity curve. Then, the program is used to apply to the physical platform. The actual velocity curve and the theoretical velocity curve will be compared to verify the idea.

MATLAB Simulation.In fig2,the planning process of type 1, 6, 7 and 8 is relatively simple, so the type 1 is only simulated, and other three types can simulated by the simulation of type 1 . Comparing type 4 with type 5, we can see that they are symmetrical with each other, and the two curve types are obtained by exchanging the values of acceleration and deceleration. Therefore, this paper only simulates type 4. In this paper, the simulation results of the four types (type 1, 2, 3 and 4 ) can represent the results of eight types of the asymmetric S-type velocity curve.For the sake of convenience, the asymmetric S-type velocity curve is simply referred to as "asymmetry" and the symmetric $\mathrm{S}$-curve is simply referred to as "symmetry".

Take $\mathrm{J}=160000 \mathrm{p} / \mathrm{s} 3, \mathrm{~A}=100000 \mathrm{p} / \mathrm{s} 2, \mathrm{~V}_{\max }=20000 \mathrm{p} / \mathrm{s}$. For the asymmetric S-type velocity curve, take $\mathrm{D}=160000 \mathrm{p} / \mathrm{s} 2$. The unit $\mathrm{P}$ of all parameters represents the pulse. There are four cases of simulation and comparison: Complete seven-velocity, no exist the constant acceleration and the deceleration section , exist the constant acceleration section but no exist the constant deceleration section, exist the constant acceleration section and the constant deceleration section.

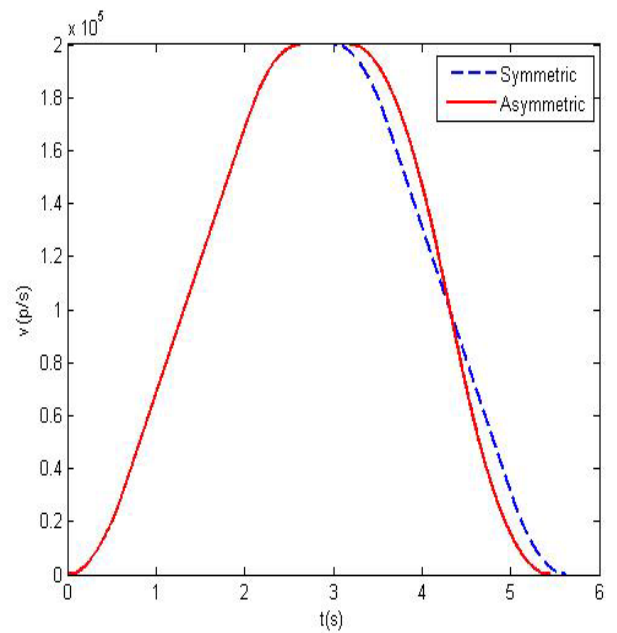

Fig3 Type 1

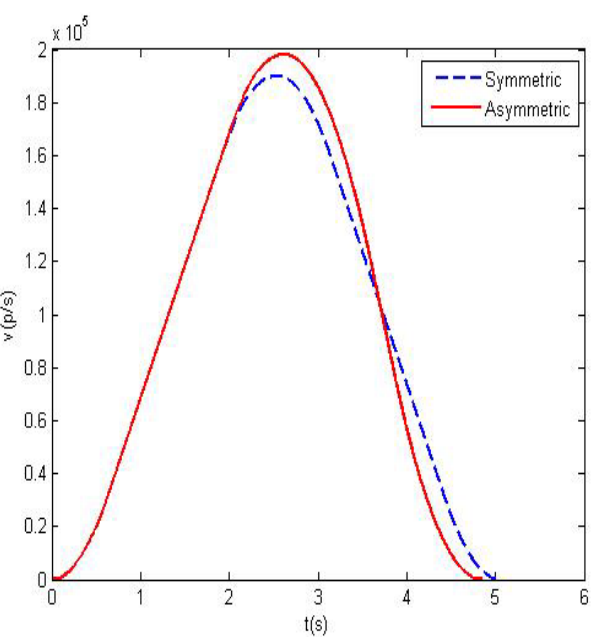

Fig4 Type 2 


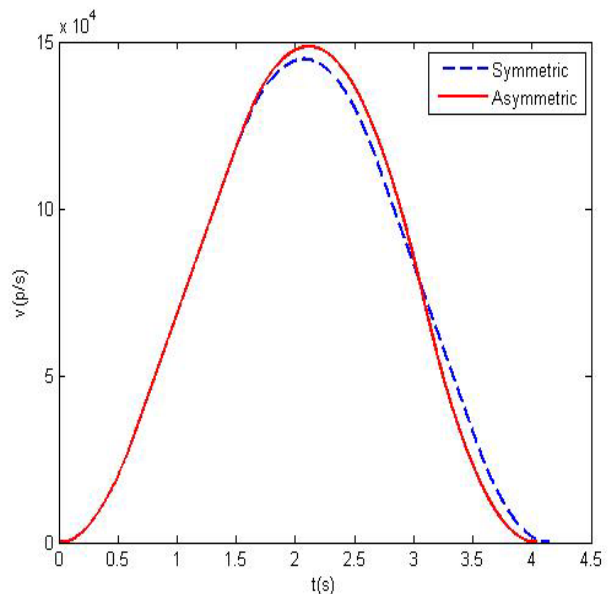

Fig5 Type 4

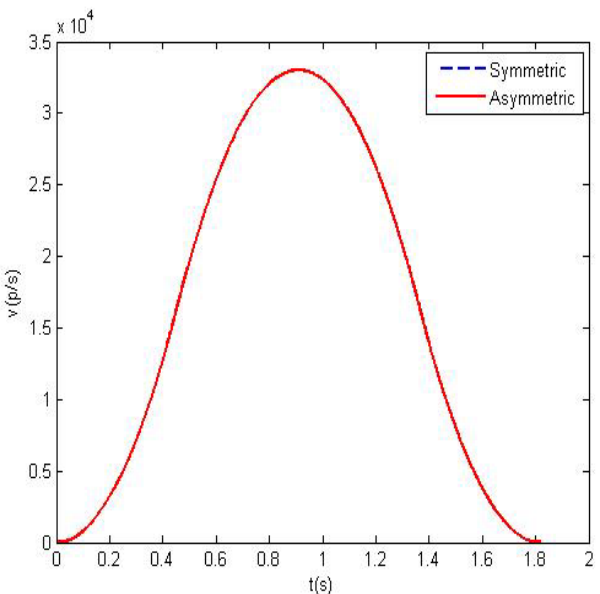

Fig.6 Type 3

1)Take $L=600000$ p. From the simulation diagram shown in Fig3, we can see that the asymmetry and symmetry are complete seven-velocity, and the actual maximum velocity is the maximum speed of the command. The both acceleration process are exactly the same, but the acceleration of the asymmetric deceleration section is larger, which lead to earlier coming to the deceleration process and faster getting to the target position than the symmetry. The time difference of the whole operation is $\Delta \mathrm{T}=5.625-5.437=0.188 \mathrm{~S}$.

2)Take $L=480000$ p. From the simulation diagram shown in Fig4, it can be seen that, the maximum velocity of the asymmetry is larger than the symmetry. The flexibility of the velocity curve at inflection point is better than the symmetry. The time difference of the whole operation is $\Delta \mathrm{T}=$ $5.05-4.84=0.21 \mathrm{~S}$ and the difference of the maximum velocity is $\Delta \mathrm{V}=198000-19000=8000 \mathrm{p} / \mathrm{s}$.

3)Take $\mathrm{L}=300000 \mathrm{p}$. As the simulation diagram shown in Fig5, it can be seen that this situation result is consistent with case 2); the asymmetric maximum velocity is larger than the symmetry, and the flexibility of the velocity curve at inflection point is also better than that of the symmetry. The time difference of the whole operation is $\Delta \mathrm{T}=4.145-4.038=0.107 \mathrm{~S}$, and the difference of the maximum speed is $\Delta \mathrm{V}=149000-145000=4000 \mathrm{p} / \mathrm{s}$.

4)Take $L=30000$ p. As the simulation diagram shown in Fig. 6, it can be seen that the asymmetric and symmetrical curves are exactly the same in this case. Maximum velocity $\mathrm{V}=$ $33000 \mathrm{p} / \mathrm{s}$, running time $\mathrm{T}=1.817 \mathrm{~S}$.

Based on the above simulation results, it can be concluded that the asymmetric S-type velocity curve has a shorter running time, smoother velocity curve at the inflection point than the symmetrical S-type velocity curve.And the asymmetric S-type velocity curve has more flexibility in the whole process and smaller impact to the motor.

Physical Verification.However, the disadvantage of the asymmetric S-type velocity curve planning is that the dichotomy used in the planning process leads to a relatively large amount of computation.With the rapid development of modern computer technology, CPU computing performance has been greatly enhanced, and fully qualified for the calculation of complex data. And its price is also lower and lower.Therefore, in order to ensure the computing ability and fast performance to satisfy the requirements, the Cortex-A9 processor+WINCE2013 embedded operating system are used as the controller in the verification algorithm, which executes position function.The controlled object adopts the combination of servo driver and servo motor, which the server driver uses the EP3E series of the MAXSINE company.And the communication between the controller and the server driver uses the EtherCAT protocol. The platform is shown by Fig.7.Finally, the algorithm is integrated into the KW company's MultiProg software to running.

The asymmetric S-type velocity curve planning process and the formulas have been given by the previous chapters in details, and the MATLAB simulation also be carried out.So the verification procedures only need to discrete the above algorithm and be expressed by the $\mathrm{C}$ language.The flow chart of program implementation is given by Fig8. 


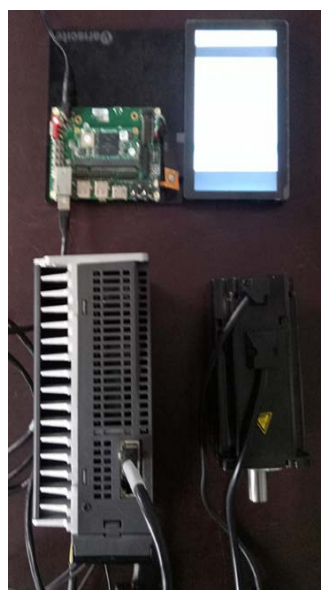

A Physical

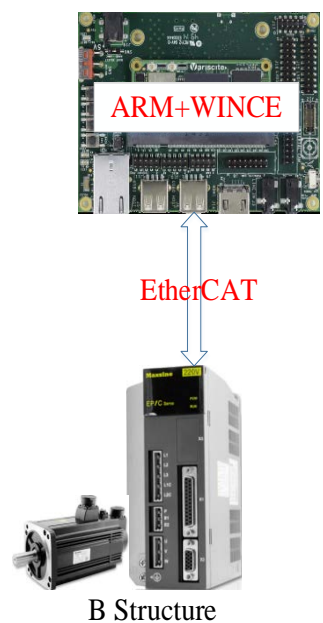

Fig.7 Verification Platform Structure

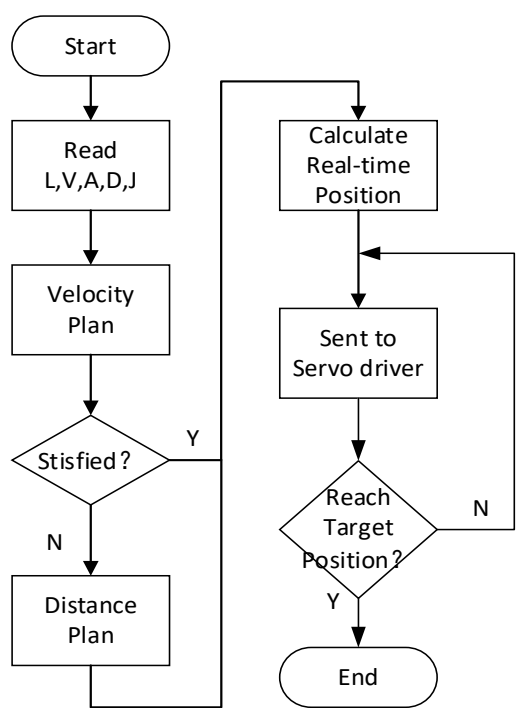

Fig.8 Program flow chart

Only at the rising edge of the command,are the velocity planning and the position planning executed and are the operating parameters of each section obtained. The real-time target position value is sent to the servo driver through the EtherCAT protocol in the process of the program loop execution.And the servo driver drives the motor according to the received real-time target position value.Therefore, the most critical of the program is to ensure get the accurate the discrete cycles. The timer accuracy of the WINDOWS system only comes to $10 \mathrm{~ms}$ level, and can not meet the requirements of motion control. So this program uses the QueryPerformanceCounter function to obtain the crystal oscillator frequency of the hardware to obtaining high precision time. This way can get microsecond time.

QueryPerformanceFrequency(\&FreqTime);//Get the crystal frequency of hardware

QueryPerformanceCounter(\&BeginTime);//Get the begin count value

QueryPerformanceCounter(\&EndTime);//Get the end count value

if $(1000 *($ EndTime.QuadPart-BeginTime.QuadPart) $>=$ FreqTime.QuadPart)//Discrete time $1 \mathrm{~ms}$

BeginTime $=$ EndTime;//Send target position to server driver

Finally, the algorithm is realized in the absolute position command, and the motor can work by calling the absolute position command and inputting the parameters in MultiProg;At the same time, the real-time velocity value of the motor can be read by the drawing software which Servo driver own. The actual velocity value is compared with the theoretical simulation value after when they were normalized in MATLAB.The comparison chart is shown by fig9. The red line is the actual velocity curve, and the blue line is the simulation velocity curve. The A/B/C/D four curves in fig9 correspond to Fig3 Fig6.
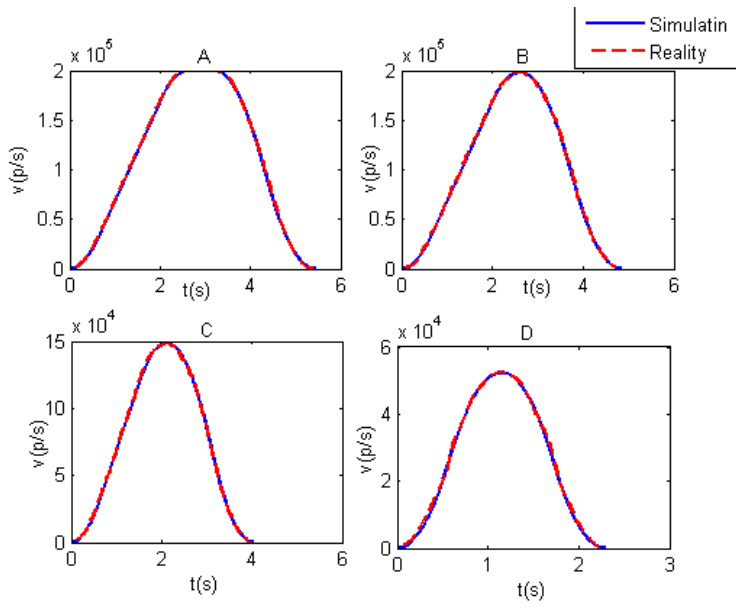

Fig9 Actual and simulation velocity curve 
It can be seen from Fig9 that the actual velocity curves and the simulation velocity curve in the four cases are coincident, which indicates that the actual situation is consistent with the theoretical analysis. The algorithm can be used to solve the asymmetric S-type curve,which has a certain practical significance and can be applied to practical situations.

\section{Summary}

First, the planning process of asymmetric S-type curve is analyzed in details.Considering the maximum velocity and the distance, the paper has analyzed all the possibility in planning of the asymmetric S-type curve. Then the symmetric S-type curve and asymmetric S-type curve are simulated in the MATLAB, and the simulation show that the asymmetric S-type curve has considerable advantages compared with the traditional symmetric S-type curve. Finally, the algorithm is applied to the actual hardware platform. By comparing the actual curve with the theoretical curve, the results are consistent with the theory. Therefore, the algorithm proposed in this paper can be used for the acceleration and deceleration planning of asymmetric S-type curve, and has a certain practical significance.

\section{Reference}

[1]CAI Rong-mao.Study on the speed curve of CNC system[J].machinery,2010,37(2): 20-23.

[2] Shi Chuan.Study on S-shape Curve Acceleration and Deceleration Control on NC System[J]. China Mechanical Engineering,2007,18(12):1421-1425.

[3] LeeC S. Generation of velocity profiles with speed limit of each axis for high-speed machining using look-ahead buffer $[\mathrm{J}]$.International Journal of Precision Engineering and Manufacturing,2010, 11(2):201-208

[4]WU Zhi-peng.S-curve Planning for Acceleration Process of Step-and-scan Lithography[J]. Modular Machine Tool \& Automatic Manufacturing Technique,2012,9:49-51.

[5]Chen Xue.Research of the S Curve Optimization Method of the Robot Acceleration and Deceleration [J].Journal of Mechanical Transmission,2013,39 (12):157-160.

[6] YANG chao.Stepper motor's acceleration and speed control based on S-curve[J].Journal Mechanical \&Electrical Engineering,2011,28(7):813-817.

[7] JIAN Yi.Research and Implementation of S Curve Acceleration and Deceleration Control for Step Motor Based on AT91SAM9261[J].Light Industry Machinery, 2012,3:62-66

[8] ZHENG Zhong-qian.Algorithm of S-shape acceleration based on control of anticipation time[J].Journal Mechanical \&Electrical Engineering, 2014,31(4):425-430.

[9] MA Yong-zhao.Study of a Five - segment S - curve Acceleration and Deceleration Algorithm[J]. Industrial Control Computer,2014, 7(12): 60,61.

[10]LIU Zhi-feng.Five-phases-curve Control Method Based on Particle Swarm Optimization[J]. Journal of Beijing University of Technology,2015,41(5):641-648

[11] Pan Haihong.A High-precision Acceleration and Deceleration Control of 7-segment Dis-symmetrical S-shape Curve for Look-ahead[J].Mechanical Science and Technology for Aerospace Engineering,2014，34(7):1024-1030 